\title{
Tangence
}

\section{Du désoeuvrement : Blanchot ou l'absence...}

\section{Florence Chazal}

Numéro 54, mars 1997

Poétique du Livre

URI : https://id.erudit.org/iderudit/025934ar

DOI : https://doi.org/10.7202/025934ar

Aller au sommaire du numéro

Éditeur(s)

Tangence

ISSN

0226-9554 (imprimé)

1710-0305 (numérique)

Découvrir la revue

Citer cet article

Chazal, F. (1997). Du désoeuvrement : Blanchot ou l'absence... Tangence, (54),

18-28. https://doi.org/10.7202/025934ar d'utilisation que vous pouvez consulter en ligne.

https://apropos.erudit.org/fr/usagers/politique-dutilisation/ 


\title{
Du désœuvrement : Blanchot ou l'absence... Florence Chazal
}

\begin{abstract}
[...] ce qui hante est l'inaccessible dont on ne peut se défaire, ce quion ne trouve pas et qui, à cause cle cela, ne se laisse pas éviter. L'insaisissable est ce à quoi l'on néchappe pas.
\end{abstract}

Maurice Blanchot ${ }^{1}$

Comment rendre compte du Livre quand celui-ci ne se livre et ne se reçoit, ne se conçoit que dans l'absence? Telle est pour ainsi dire l'épreuve, ou l'impasse, dans laquelle Blanchot s'engage avec L'espace littéraire, qu'il présente en exergue comme "un livre d'éclaircissement". Car, *l'autre nuit*, nul ne peut s'en saisir, nul ne peut s'y tenir. Telle fut l'expérience d'Orphée aux Enfers. Blanchot fait donc appel au mythe afin d'illustrer ce rapport impossible qui lie l'écrivain, ou le lecteur, à l'oeuvre (le Livre) et qui conduit, inévitablement, au désœuvrement.

Il ne s'agira donc pas ici de faire apparaître le Livre par quelque tour de magie. Le pari est perdu d'avance. Nous ne ferons qu'esquisser les grandes lignes d'une pensée aussi complexe que celle de Blanchot. Nos propos s'annoncent d'emblée réducteurs. Avancer cela ne procède pas uniquement d'une simple précaution écrite puisque, comme nous le montre Blanchot, tout discours est, par définition, réducteur car l'objet, lui, reste irréductible. Par conséquent, nous nous en tiendrons à proposer quelques pistes de lecture pour ceux et celles qui s'aventurent aux abords de l'espace littéraire où *les contradictions ne s'y excluent pas, ne s'y concilient pas" (p. 27). Blanchot nous répondrait sans doute que nul n'est besoin de se prémunir, car l'on sort toujours démuni. Jusqu'où pouvons-nous le suivre?

1 Maurice Blanchot, Lespace littéraire, Paris, Gallimard, coll. "Folio Essais ", [1955] 1988, p. 348. Toutes les références ultérieures renvoient à cette édition; les pages seront signalées entre parenthèses. 


\section{D'une parole intransitive}

Bien que Blanchot ne le pose pas explicitement, la théorie du langage qu'il développe à la suite de Mallarmé constitue le fonclement de sa conception de la littérature et de la création. Il reprend ainsi la distinction de Mallarmé entre "parole brute", d'une part, et "parole essentielle" ou apoétique», d'autre part ${ }^{2}$. Il rappelle que dans les deux cas, et selon la logique même du langage, le mot entraîne la disparition de la chose, c'est-à-dire qu'il la représente dans son absence, et ne désigne, finalement, que l'absence.

Les mots, nous le savons, ont le pouvoir de faire disparaître les choses, de les faire apparaitre en tant que disparues, apparence qui n'est que celle d'une disparition, présence qui, à son tour, retourne à l'absence (p. 45).

Or - et c'est ici que réside la différence - la parole "brute" se donne à entendre comme "immédiate", puisqu'en elle "le langage se tait comme langage" (p. 40) afin de re-présenter - de rendre présent - ce à quoi elle réfère. La parole brute correspond en somme au discours d'usage (à entendre dans les différents sens du terme). Elle sert ainsi à nous mettre en rapport avec le monde, à construire des ponts. Instrumentale, elle constitue "un outil dans un monde d'outils" (p. 40). Elle est utile car elle permet au sujet de s'ancrer et de se positionner dans le monde. Elle génère un sentiment d'appartenance et de sécurité associé à - la familiarité du lieu natal "(p. 41). Elle donne à croire. Elle fait illusion, au sens de praxis, puisque la perception ainsi produite du monde comme "abri * (p. 42) ne se réduit qu'à son -apparence stable. (p. 40, nous soulignons). La parole brute produit du semblant.

Tandis que, dans l'autre parole, a le langage parle comme essentiel [...] les mots, ayant l'initiative, ne doivent pas servir à désigner quelque chose ni à donner voix à personne, mais [...] ont leurs fins en eux-mêmes. Désormais [...] le langage se parle" (p. 42). Ainsi, la parole essentielle, ou poétique, ne renverrait pas au monde mais serait intransitive. Il s'agit d'une parole "pure" qui ne fait qu'accomplir son "acte d'autodestruction" (p. 45), à

2 Peu de pages sont consacrées à établir cette distinction (p. 38-43) qui nous semble néanmoins fondamentale puisqu'elle inaugure toute une série d'oppositions qui finissent par creuser un écart infranchissable entre - le monde d'une part, et - l'espace littéraire - d'autre part. 
l'infini - de la même façon qu'un signifiant ne renvoie qu'à un autre signifiant. Le référent est évacué, ou plutôt évidé. La parole essentielle ne re-présente rien mais (se) présente (dans) son absence.

\section{De l'objet, ou de son absence}

De cette première distinction, posée ici comme fondatrice, dérive celle qui sépare l'ouvre du livre. Celui-ci ne constitue que "le substitut, l'approche et l'illusion" (p. 16) de l'weuvre. Le livre, dans sa matérialité historique et culturelle, se range donc du côté de la parole brute, du langage "ordinaire *. Il n'est qu'effet d'œutre, effet de signifié, seul objet disponible et malléable: un leurre. Car * l'œuvre - l'œuvre d'art, l'œuvre littéraire - n'est ni achevée ni inachevée: elle est. Ce qu'elle dit, c'est exclusivement cela : qu'elle est - et rien de plus. En dehors de cela, elle n'est rien. [...] Elle est sans preuve, de même qu'elle est sans usage" (p. 14-15). Elle est, essentielle, mais ne consiste pas. Elle est rien. En elle, la parole essentielle, "le langage se parle, le langage comme ceuvre et l'cuvre du langage" (p. 42). L'wuvre serait donc "pur " langage, "murmure, " ressassement ", "clapotement", etc., de toutes les façons, insaisissable.

\section{Personne: de l'écrivain}

Par conséquent, l'écrivain ne peut chercher qu'à "se faire l'écho de ce qui ne peut cesser de parler, - et, à cause de cela, pour en devenir l'écho [...] lui imposer silence" (p. 21, nous soulignons). Réitérer l'absence, telle est, pour l'écrivain, l'unique façon de participer de l'ouuvre, sans ne jamais pouvoir se l'approprier. Car elle a exige de l'écrivain qu'il perde toute "nature", tout caractère $[. .$.$] devienne le lieu vide où s'annonce l'affirmation$ impersonnelle" (p. 61). Écrire, c'est donc passer du "Je * au * $\mathrm{Il}$ " du personnel à l'impersonnel - , comme le dit Blanchot en paraphrasant Kafka (p. 31). Écrire, c'est devenir personne.

À l'approche de l'œuvre, l'écrivain est amené à abandonner toute identité et toute autorité. Il doit s'abandonner, car

l'artiste qui s'offre aux risques de l'expérience qui est la sienne, ne se sent pas libre du monde, mais privé du monde, non pas maître de soi, mais absent de soi, et exposé à une exigence qui, 
le rejetant hors de la vie, louvre à ce moment où il ne peut rien faire et où il n'est plus lui-même (p. 57-58). ${ }^{3}$

Ex-stasis et aphanisis. L'écrivain est tour à tour altéré rendu autre - et desti-tué.

L'écrivain ne participe de l'œuuvre que de par son propre effacement: "celui qui écrit l'oeuvre est mis à part, celui qui l'a écrite est congédié " (p. 14) - sans même le savoir. Cet insu, condition du désir, ne mène qu'au *désceuvrement ", à l'absence d'œuvre, et oblige l'écrivain à se remettre à l'xuvre. "Écrire est maintenant l'interminable, l'incessant" (p. 20). Constamment maintenu à distance ou à l'écart de l'ouuvre, l'écrivain non seulement " ne lit jamais son ceuvre. (p. 17) mais "ne terminant son cuvre qu'au moment où il meurt, ne la connaît jamais" (p. 16). Seul reste le livre, demeure vide.

\section{Personne : du lecteur}

Or, "[q]u'est-ce qu'un livre qu'on ne lit pas? Quelque chose qui n'est pas encore écrit" (p. 254). Une fois l'écrivain éconduit, c'est au tour du lecteur de prendre le flambeau et de arendre l'œuuvre à elle-même, à sa présence anonyme, à l'affirmation violente, impersonnelle qu'elle est" (p. 254). Si l'interdit posé à l'écrivain - "Noli me legere" (p. 17) - s'oppose à l'appel lancé au lecteur - "Lazare, veni foras" (p. 257) - écrivain et lecteur occupent une position symétrique, voire iclentique, face à l'œuvre ${ }^{4}$. En effet, le lecteur "est lui-même toujours foncièrement anonyme, il est n'importe quel lecteur, unique, mais transparent" (p. 254), livré, lui aussi, à l'impersonnel. "Ce qui menace le plus la lecture: la réalité du lecteur, sa personnalité, son immodestie, l'acharnement à vouloir demeurer lui-même en face de ce qu'il lit" (p. 263, nous soulignons). Le lecteur doit renoncer à tout ancrage, à toute assise: au monde. Il faut qu'il s'efface pour permettre à l'weuvre de a s'affirmer comme chose sans auteur et aussi sans lecteur" (p. 255). Le lecteur vient en quelque sorte confirmer l'absence de l'auteur par sa propre absentéisation. C'est pourquoi leurs fonctions sont à la fois symétriques et complémentaires -

3 C'est pourquoi, selon Blanchot, seul l'écrivatin qui pose sa plume se soustrait a la fascination de l'ceuvre et se trouve alors en position de mâttrise (p. 19).

4 - Auteur et lecteur sont à égalité devant l'oeuvre et en elle *, concluera l3lanchot (p. 302). 
complémentaires car écrivain et lecteur s'éclipsent l'un l'autre. Semblables car ils sont personne.

À la mise en ceuvre du premier qui aboutit au désceuvrement succède la mise en ouvre du second qui retombe aussi finalement dans le désceuvrement. En effet, Blanchot le souligne, l'expérience de l'écrivain et du lecteur est toujours première face à l'outuvre qui, elle, ne cesse de se dérober. Tout n'est donc que commencement et recommencement. Pouvoir et possibilité liés au commencement se transforment inéluctablement en impouvoir et impossibilité - qui mènent à un recommencement.

\section{Sans fin ni commencement}

Cette succession temporelle de moments contraires qui prennent "la forme personnifiée du lecteur et de l'auteur" (p. 264) ne fait que représenter l'antagonisme atemporel inhérent à l'ceuvre, - l'intimité et la violence de mouvements contraires qui ne se concilient jamais et qui ne s'apaisent pas, tant du moins que l'œuvre est ceuvre" (p. 300). L'ouvre est - sur le mode de n'être pas c'est-à-dire "est aussi toujours en même temps l'un et l'autre, l'entrelacement du Oui et du Non ${ }^{5}$, le flux et reflux cle l'ambiguité essentielle" (p. 321).

Le rôle de l'écrivain et du lecteur consiste donc non pas à faire de l'cueuvre un objet fini mais, au contraire, à assurer et à maintenir son constant renouvellement. Écrivain et lecteur doivent se désincarner, faire le mort, afin d'animer "le vide cadavérique" (p. 257) de l'ouvre - la re-susciter ${ }^{6}$. Apparition-disparition, fort-da, l'ceuvre ne cesse de se donner et de se reprendre, de se faire et de se défaire: danse macabre ${ }^{7}$.

L'image du cadavre, à la fois présent et absent, est peut-être la seule qui nous sera offerte de l'ouvre. Comme l'indique Blanchot, "la présence cadavérique établit un rapport entre ici et

5 On peut voir, dans ce Oui, le "Lazare treni forasw aldessé au lecteur et, dans ce Non, le "Noli me legere. imposé à l'auteur.

6 Image christique de l'ceuvre qui s'énonce dans le - Lazare veni foras. et le - Noli me legere.

7 Nous reprenons ici l'image utilisée par 13lanchot pour parler de la lecture: "(...) une danse avec un partenaire invisible dans un espace séparé, une danse joyeuse, éperdue, avec le "tombealu" (p. 261) - tombealu vide (clu Christ?), clemeure qui n'abrite rien. 
nulle part" (p. 344), entre ce qu'il nomme le monde d'un côté et l'espace littéraire de l'autre, à savoir l'espace de la mort, les Enfers où Orphée a laissé Eurydice. En effet, le sujet blanchotien entretient un rapport similaire à l'oeuvre et à la mort. Car si l'être humain est mortel, il ne peut néanmoins se rendre maittre de sa propre mort - dire "je meurs" ou bien a je suis mort" "On meurt", uniquement, dans l'anonymat (p. 323). Le reste est simulacre $^{8}$. Or, le cadavre n'est-il pas lui non plus res-semblance? De la mort, l'on ne peut rien savoir, de l'ouuvre non plus. Quand l'cuuvre se dévoile, elle continue à se dérober... L'on finit toujours ainsi par buter sur l'indêterminé et sur l'illimité.

Il s'agit donc non pas de ramener l'ceuvre à soi mais de la rendre à elle-même, c'est-à-dire au vide dont elle se constitue.

Qui n'appartient pas à l'ouuvre comme origine, qui nalppartient pas à ce temps autre où locuvre est en souci de son essence, ne fera jamais ceuvre. Mais qui appartient à ce temps autre, appartient aussi à la profondeur vide du désceuvrement où de l'être il n'est jamais rien fait. (p. 51)

L'oeuvre ne cesse à la fois de s'originer et de rechercher sans fin son origine: "ce qui doit parler dans l'ceuvre, c'est son origine" (p. 321), ce qui, exclu, crée la structure. L'origine reste donc toujours plus antérieure à elle-même, "car c'est le propre de l'origine de toujours être voilée par ce dont elle est l'origine" (p. 314). L'écrivain opère en quelque sorte une fonction de coupure coupure, commencement tout à fait relatifs qui ne peuvent se confondre avec l'origine. Car l'origine est toujours à jamais perdue. Donc, "ce qui est premier, ce n'est pas le commencement, mais le recommencement, et l'être, c'est précisement l'impossibilité d'être une première fois" (p. 327). Comme le dit Blanchot, l'cueuvre est et ne dit que cela, l'oeuvre est parole essentielle, ressassement "éternel", Oui et Non à la fois, dévoilement et voilement. C'est pourquoi l'œuvre "ne tranche ni ne surgit mais revient" (p. 326). Et l'on pourrait ajouter que si elle revient, c'est toujours à la même place, sous la forme d'un rendez-vous manqué. L'ócuvre ne cesse pas de ne pas s'écrire. Car elle est, par définition, (l')impossible.

Abîme sans fond, *lumière qui est aussi l'abîme, une lumière où l'on s'abîne, effrayante et attrayante", tout comme "la Mère"

8 Blanchot semble ranger le suicide dans cette catégorie : te suiciclé ne donnerait que l'illusion cle sé rendre maître de sa propre mort (p. 134). 
qui fascine tant l'enfant - la Mère, seule figure humaine bien vite assimilée à "l'immense Quelqu'un sans figure. (p. 30). Ainsi celle qui tient lieu de l'origine et vient la figurer, lui donner figure, la Mère est, elle aussi, absorbée par l'Impersonnel. Manque l'origine.

\section{"L'horreur du vide"}

C'est donc ici, dans le questionnement des conditions de possibilité de l'œuuvre, à sa naissance insaisissable, que se situe Blanchot le critique, la critique de Blanchot, "en dehors de toute signification, de toute affirmation historique, esthétique" (p. 48). Il s'agit de cerner au plus près et de préserver "cette manière de lire, présence à l'ouuvre comme à une genèse" (p. 269), genèse sans cesse rejouée - partie remise.

"L'ceuvre d'art n'est jamais liée au repos, elle n'a rien à voir avec la tranquille certitude qui rend coutumiers les chefs-d'veuvre, elle ne s'abrite pas dans les musées. (p. 271, nous soulignons). L'ceuvre ne fait pas partie de ce monde où circule la parole brute. Nous retrouvons en effet dans les éléments soulignés les attributs de ce monde *illusoire "qu'écrivain et lecteur sont forcés d'abandonner quand ils sont confrontés à la parole essentielle. C'est pourquoi Blanchot s'élève contre les pratiques de celui qu'il nomme le *lecteur spécialiste", qui ne sait pas se taire: "L'horreur du vide se traduit ici par le besoin de le remplir avec un jugement de valeur" (p. 268), c'est-à-dire de l'intégrer à un système, et finalement de l'assimiler au monde. Blanchot remet donc en question la. notion même de communauté interprétative. Le fondement de toute institution littéraire en est ainsi ébranlé. L'institution littéraire n'est qu'un monument vide, "musée", "tombeau", qui s'efforce néanmoins à masquer le vide qu'elle ne peut résorber. À l'encontre de ces tentatives de réification systématiques, Blanchot prône le désaisissement et le désistement. Il dit se refuser à combler ce vide qui doit être préservé car il est ce qui permet le jeu et ce qui constitue l'enjeu, "accomplissement du visible en l'invisible dont nous avons la charge. ( $p$. 181). Il illustre ce mouvement par le biais de la figure - emblématique - d'Orphée qu'il situe au centre de L'espace littéraire". La démarche d'Orphée s'avère exem-

9 lilanchot nous donne en exergue de Lespace littéraire le sens de cette plongée dans l'abîme: - Un livre, même fragmentaire, a un centre qui l'attire : [...] ici, vers les pages intitulées "Le regard d'Orphée" 
plaire, car elle vient à désigner ale point central de l'œuvre [qui] est l'œuvre comme origine, celui que l'on ne peut atteindre, le seul pourtant qu'il vaille la peine d'atteindre " (p. 60).

Orphée est allé chercher Eurydice aux Enfers. Il ne peut la ramener au jour qu'à la condition de ne pas la regarder. Il désobéit et elle disparaît. Le mythe devient une véritable allégorie parabole - et mise en abyme du sort réservé à celui qui s'aventure aux abords de ce que Blanchot nomme "l'autre nuit", "la nuit sacrée" (p. 231). L'espace littéraire constitue cette "région qu'on ne peut amener à la lumière "(p. 28), tout comme Orphée ne peut sortir Eurydice des ténèbres - Eurydice qui représente "l'extrême que l'art puisse atteindre. (p. 225). Et si celui qui s'approche de l'outure ne peut se l'accaparer, Orphée ne peut non plus re-garder Eurydice: * le détour est le seul moyen de s'en approcher" (p. 225).

"L'erreur d'Orphée semble être alors dans le désir qui le porte à voir et à posséder Eurydice, lui dont le seul destin est de la chanter " (p. 227). Or, "Orphée n'avait fait qu'obéir à l'exigence profonde de l'ouuvre. (p. 228) quand il a détruit ce qu'il venait chercher. L'œuvre impose une double contrainte: s'approcher et se détourner, s'approcher pour se détourner. Cette nécessité paradoxale constitue bien la teneur de toute relation à l'outuvre. L'un exige la disparition de l'autre: l'un ou l'autre. Telle est, selon Blanchot, la logique de la condition de celui qui se consacre à l'art: "il y a aux environs de l'art un pacte noué avec la mort, avec la répétition et avec l'échec" (p. 326) et avec le silence. Si Blanchot fait appel au mythe afin de mettre en scène le rapport impossible qui noue le sujet à l'œuvre, il ne s'agit pas cependant de revenir à un temps mythique d'avant la séparation. Il s'agit au contraire d'illustrer l'écart insurmontable qui rend l'ouvre inaccessible, de rejouer en somme le drame de la séparation et de la perte.

\section{La figure du passeur}

Blanchot opère un détournement similaire à celui qu'il met en scène par le biais de la figure d'Orphée quand il s'efface pour laisser parler les écrivains et poètes chez lesquels il reconnaît une fraternité de pensée qu'il nomme "malheur de l'égarement "ou "migration féconde" (p. 332). Plutôt que d'analyser ou d'interpréter leurs oeuvres litteraires, Blanchot se penche sur le journal 
26

intime des écrivains qui nourrissent sa réflexion et parcourent les pages de L'espace littéraire (parmi eux Mallarmé, Kafka, Rilke, Hölderlin). Il ne s'agit cependant nullement de ranimer ceux-ci sous la forme d'une biographie. Si le journal - "mémorialn permet de conserver "un nom" et d'inscrire "une date "afin d'échapper à l'oubli de soi et du quotidien, sacrifice exigé par l'ouvre, paradoxalement, "le moyen dont se sert l'écrivain pour se rappeler à soi, c'est [...] l'élément même de l'oubli : écrire" (p. 24). $\mathrm{Si}$ arrêter d'écrire permet de restaurer le temps, tenir un journal constituerait une tentative de retrouver le temps, perdu dans l'acte d'écriture ${ }^{10}$. Quête insensée et dérisoire mais qui témoigne cependant du "mouvement même de l'expérience d'écrire, au plus proche de son commencement [...]. C'est sous cette perspective que le Joumal ${ }^{11}$ doit être lu et interrogén (p. 63-64). Il est d'ores et déjà évident que Blanchot s'intéresse à la démarche des écrivains dont il côtoie les textes. C'est la question et non la réponse, le processus et non le résultat qui importe - ${ }^{\circ}$ comme si renoncer à échouer était beaucoup plus grave que renoncer à réussir n (p. 229). Dans ce risque sans cesse renouvelé se trouve "la source de toute authenticité (p. 229). Ainsi, quand il reprend la question de l'oulvre, conclut-il: " $\grave{A}$ cette question, il ne peut être répondu. Le poème est l'absence de réponse. Le poète est celui qui, par son sacrifice, maintient en son ceuvre la question ouverte" (p. 332).

Blanchot cherche, toujours et encore, à retracer au plus près de sa source les conditions de possibilité de l'œuuvre, c'est-à-dire * le point où ici coïncide avec nulle part* (p. 52). Point fuyant, point de fuite: "point sacrificiel" (p.184) où l'écrivain finit par sacrifier à la fois le monde et l'ouuvre. L'écrivain, "arpenteur" (p. 94), "migrateur" (p. 319), est interminablement condamné à l'errance et à l'erreur: "Erreur signifie le fait d'errer, de ne poutvoir demeurer parce que, là où l'on est, manquent les conditions d'un ici décisif "(p. 319, nous soulignons), c'est-à-dire du monde. Perpétuel exilé, l'écrivain parcourt sans fin le désert en quête de la Terre Promise (p. 82), c'est-à-dire de l'œeuvre. Se retrouve la distinction, posée au début de notre incursion aux abords de l'espace littéraire, entre parole brute et parole essentielle. Le sujet

10 "Écrire, c'est se livrer à la fascination de l'absence de temps. (p. 25), c'està-clire - un temps sans négation. (p. 26). Le monde temporel s'oppose ainsi à l'espace (littéraire) atemporel.

11 Il s'agit ici du Joumal de Kafka. 
blanchotien, qu'il soit écrivain ou lecteur, doit renoncer à "la familiarité du lieu natal" (p. 41), figure de l'origine, point de repère dans le désert. Car dans le désert, toute trace s'efface. Celui qui se risque à l'approche de l'oeuvre est amené à quitter le monde comme "abri . Il est livré au "Dehors", à "l'Ouvert", au Livre ainsi grand ouvert: le Hors Livre. Il se trouve en quelque sorte dans les limbes. Face à cette impasse, le seul rôle qu'il puisse tenir est celui de passant ou de passeur, tout comme Orphée ne peut que chanter Eurydice - chant du cygne:

C'est que nous ne sommes pas seulement parmi ceux qui passent, mais, dans ce royaume du penchant, nous sommes aussi ceux qui consentent à passer, qui disent Oui à la disparition et en qui la clisparition se fait dire, se fait parole et chant (p. 193).

Cette posture tient à la fois de la mystique et de la prophétie. Il s'agit, par effacement de soi, de laisser parler en soi l'éternel Absent, de l'incarner, pour un temps, dans "l'isolement prophétique qui, en deçà du temps, annonce toujours le commencement " (p. 333) ${ }^{12}$. Il faut préciser que dans l'univers blanchotien n'existe aucun espoir d'union (mystique) ou de rédemption. Seul subsiste le tragique de la répétition. Car "[l]es dieux aujourd'hui se détournent, ils sont absents, infidèles, et l'homme doit comprendre le sens sacré de cette infidélité divine, non pas en la contrariant, mais en l'accomplissant pour sa part. (p. 367).

\section{$\stackrel{* *}{* *}$}

Blanchot, on l'aura compris, s'élève contre une conception de la littérature qui valorise l'objet produit au détriment de l'expérience - première - de l'écrivain ou du lecteur confronté à la parole essentielle. Il souligne ainsi le double leurre associé à l'objet. L'objet, d'une part, n'est qu'un leurre, une illusion quand nous croyons le trouver et, d'autre part, il fonctionne comme un leurre en ce qu'il pousse l'écrivain à écrire, à continuer d'écrire. C'est pourquoi l'objet littéraire, le livre, est toujours à venir. Blanchot illustre bien cette dynamique nécessaire quand il souligne le fait qu'Orphée ne devait pas retrouver Eurydice. La retrouver serait trouver la mort, la mort du désir et donc du sujet.

12 Ces mots sont les derniers de Lexpace littêraire, en excluant les annexes. 
L'écrivain ne peut qu'approcher l'espace littéraire, rester à ses abords, et faire ainsi bord à l'œuvre. C'est pour lui la seule manière de participer de l'œuvre dont il reste exclu - exilé et désoeuvré. L'on ne peut s'empêcher ici de penser à l'image que Lacan reprend de Heidegger, celle du potier qui tout en façonnant les bords de son vase crée le vide central qui l'occupe (la Chose). Cette dimension sublimatoire qui, selon les mots de Lacan, "élève l'objet à la dignité de la Chose "13, Blanchot la passe sous silence.

D'un autre côté, l'on peut se demander si le refus d'objectiver l'ueuvre ne cède pas finalement à une sacralisation ${ }^{14}$ ou à une fétichisation du Rien (de la Chose). Que gagner à poser la perte comme infini(e), sinon son évitement même, évitement du deuil? N'est-ce pas alors courir le risque de tomber dans la jouissance, celle de l'ab-sens? - image de la jouissance que Blanchot nous présente par le biais d'un écrivain qu'il dépeint livré à la passivité, proche de l'évanouissement. Mais l'on pourrait aussi voir dans ce drame sans cesse rejoué de la séparation et de la division, de la perte et du manque, un moyen de l'exorciser ou de l'apprivoiser. L'écrivain joue de la perte: il la remet sur le tapis en quelque sorte, la met en scène.

Comment donc rendre compte du Livre, de l'œuvre? Si Blanchot ne fait que souligner le versant a négatif " du travail de l'œuvre, sa structure en creux, ne transmet-il pas (malgré lui?) un savoir (inconscient?) sur l'impossible de l'œuvre? De même, Blanchot s'efforce-t-il de se détourner, de tourner le dos au travail de l'imaginaire ${ }^{15}$ quand il construit l'image d'un écrivain ou met en scène un lecteur qui se trouvent, chacun, dans une solitude absolue et connaissent une perte d'identité quasi totale. Ne passet-il pas ainsi sous silence l'weuvre de l'imaginaire, l'imaginaire à l'ouvre qui lui permet de dialoguer avec d'autres écrivains, de les lire et de les commenter et de former une communauté? - aussi inavouable soit-elle pour lui ${ }^{16}$ ?

13 Jacques Lacan, Le séminaire, livre VII: L'éthique de la psychanalyse. Paris, Seuil, coll. "Le champ freudien-, 1986, p. 133. Voir alussi le chapitre intitulé «De la création ex nibili., p. 139-152.

14 - Le sacré est ce vide même, ce pur vide de l'entre-deux qu'il faut maintenir pur et vide. (p. 330).

15 L'imaginaire est ici à comprendre comme le registre lacanien de l'identification et du leurre et peut s'apparenter à la parole brute.

16 Nous faisons ici bien entendu référence à l'essai de Blanchot qui s'intitule $L a$ communauté inatxuable, Paris, Minuit, 1983. 